\title{
Expression of $N$-Acetylgalactosaminyltransferase-6 Is Related to Expression of Cell Adhesion Molecules in Endometrial Cancer
}

\author{
TOMOKO KURITA ${ }^{1}$, THUY NGUYEN THI ${ }^{1}$, CHIHO KOI $^{1}$, MIDORI MURAKAMI $^{1}$, \\ SEIJI KAGAMI ${ }^{1}$, HIROTO IZUMI ${ }^{2}$ and TORU HACHISUGA ${ }^{1}$ \\ Departments of ${ }^{1}$ Obstetrics and Gynecology, and ${ }^{2}$ Occupational Pneumology, \\ University of Occupational and Environmental Health, School of Medicine, Kitakyushu, Japan
}

\begin{abstract}
Background: The aberrant glycosylation of mucin type O-glycans is thought to be associated with functional alteration of cancer cells, including adhesive properties, as well as their potential for invasion and metastasis. Positive expression of $\mathrm{N}$-acetylgalactosaminyltransferase-6 (GalNAcT6) may also be a marker for aberrant O-glycans in carcinogenesis. We previously reported that over-expression of GalNAc-T6 had a strong association with endometrial cell invasion ability in vitro. Materials and Methods: This study investigated the relationship between GalNAc-T6 expression and cell adhesion molecules in 218 endometrial carcinomas by immunohistochemistry. Results: Expression of GalNAc-T6 was found to be significantly related to expression of $E$ cadherin. Positive expression of GalNAc-T6 was significantly associated with better histological grade and good clinical prognosis of patients, but positive E-cadherin and $\beta$-catenin expression were not significantly associated with improved overall survival. Conclusion: GalNAc-T6 might be related to cell-cell adhesion in the early phase of cancer invasion in endometrial carcinoma.
\end{abstract}

Endometrioid endometrial carcinoma is the most common gynecological carcinoma in developed countries including Japan $(1,2)$. Recent studies have documented that cell invasion during tumor progression may be critically dependent on the process of epithelial-mesenchymal transition (EMT) (3-7). Disassembly of cell-cell junctions together with down-regulation of epithelial protein E-

Correspondence to: Tomoko Kurita MD, Department of Obstetrics and Gynecology, University of Occupational and Environmental Health, School of Medicine, 1-1 Iseigaoka, Yahatanishi-ku, Kitakyushu, Fukuoka, 807-8555, Japan. Tel: +81 936917449, Fax: +81936919337, e-mail: t-kurita@med.uoeh-u.ac.jp

Key Words: $O$-Glycosylation, polypeptide $N$-acetylgalactosaminyltransferase- 6 , endometrial carcinoma, cell adhesion. cadherin is an important hallmark of EMT. Decreased Ecadherin expression in endometrial carcinoma has been shown to be associated with poor differentiation and deep myometrial invasion (8). $\beta$-Catenin expression associated with loss of E-cadherin expression was found to be involved in the acquisition of aggressive biological behavior (9). The change of expression of cell adhesion molecules plays an important role in the progression of endometrial cancer.

It is well known that malignant transformation and cancer progression are related to alteration in cell-surface carbohydrate antigens, as well as aberrant glycosylation (1012). $N$-Acetylgalactosaminyltransferases-6 (GalNAc-T6) is thought to be a marker for aberrant glycosylation (13). Aberrant expression of GalNAc-T6 has been reported to have a prognostic role in several different kinds of cancer (14-18). There are reports that change in GalNAc-T6 expression causes morphological change accompanied by the cadherin switch (19) and EMT (4-6). However the molecular mechanism explaining the relationship between GalNAc-T6 status and local invasion or metastasis in endometrial carcinoma remains to be elucidated.

In the present study, we investigated the expression of GalNAc-T6 in endometrioid endometrial carcinoma and expression of E-cadherin.

\section{Materials and Methods}

Patients and tumor samples. Two hundred and eighteen patients with endometrioid endometrial carcinoma were treated at the University of Occupational and Environmental Health Hospital in Kitakyushu, Japan, between 1990 and 2009. All patients with available follow-up data comprised the cohort of this retrospective study. The patients received standard treatment for endometrial carcinoma with hysterectomy and bilateral salpingo-oophorectomy, pelvic lymph node dissection, para aortic lymph node dissection or biopsy; subsequent chemotherapy was decided according to surgical stage and classification of risk factors. Paclitaxel-based chemotherapy consisted of paclitaxel $\left(180 \mathrm{mg} / \mathrm{m}^{2}\right)$ and carboplatin (area under the curve $=5$ ) on day 1 with a 3 -week interval without drug administration in 3-6 courses. The grade of tumors was 
determined according to the International Federation of Gynecology and Obstetrics (FIGO) classification of endometrial cancer (20). The study was established based on the agreement of the Review Board of the Hospital on Ethical Issues (H27071).

Preparation of antibodies against GalNAc-T6. Polyclonal antibody to GalNAc-T6 was raised by multiple immunizations of New Zealand White rabbits with synthetic peptides (Figure 1). The synthetic peptide sequence of the $C$-terminal of GalNAc-T6 was GFYT-PAELKPFWERPPQDP. The specificity of antibody was confirmed by western blotting and immunohistochemistry with peptide competition as described previously (6).

Immunohistochemistry. Histological sections $4 \mu \mathrm{M}$-thick were cut from formalin-fixed, paraffin-embedded tissues for immunohistochemistry. The samples were deparaffinized in xylene, then rehydrated through graded ethanol, and washed in phosphate-buffered saline (PBS) Antigen retrieval proceeded at $121^{\circ} \mathrm{C}$ for 3 min with $0.01 \mathrm{M}$ citrate buffer $(\mathrm{pH}=6.0)$. Endogenous peroxidises were quenched by Peroxidase Blocking Regent (Dako, Kyoto, Japan) for $5 \mathrm{~min}$, then sections were washed in PBS for 3 minutes. The sections were then incubated with polyclonal antibody to GalNAc-T6 $(1: 1,600)$ for 1 hour at room temperature, mouse monoclonal antibody to E-cadherin (diluted 1:350; CST signaling, Tokyo, Japan) at $4^{\circ} \mathrm{C}$ overnight, and mouse monoclonal antibody to $\beta$-catenin (diluted 1:800; BD Bioscience, San Jose, CA, USA) at room temperature for $25 \mathrm{~min}$. Antibody biding was visualized using the Envision+ Dual link system (DAKO, Kyoto, Japan). Finally slides were counterstained with hematoxylin and mounted. Samples of well-differentiated tubular pancreatic adenocarcinoma were used as positive controls for GalNAc-T6 (15). The negative controls were stained after omitting the experimental step of incubation with primary antibody. Immunostained slides were analyzed independently by two authors without knowing patient outcomes. Cytoplasmic GalNAc-T6 expression and apical membranous E-cadherin and $\beta$-catenin expressions were graded into four categories. A score was obtained for the area showing the strongest staining intensity, ranging from 0 to 3 in which 0 was no staining and 3 was defined as strong staining. In order to analyze the significance of expression patterns, staining score was combined into negative (score 0 and 1 ) and positive (score 2 and 3) groups (Figure 2).

Statistical analysis. Statistical analysis was carried out using SPSS version 18.0 (SPSS Inc, Chicago, IL, USA). Mann-Whitney $U$-test (Pearson $\chi^{2}$ ) was used to assess the relationship between clinicopathological variables and expression of the three antigens. Kaplan-Meier method with log-rank test, and Cox proportional hazards models were used to evaluate the prognostic role of protein expression. A value of $p<0.05$ was considered to be statistically significant.

\section{Results}

Clinicopathological characteristics. The study included 152 patients with stage I disease $(69.8 \%), 12$ with stage II (5.5\%), 50 with stage III $(22.9 \%)$, and four with stage IV disease $(1.8 \%)$. The grade of the tumors was determined according to the FIGO classification of endometrial cancer (20): 127 patients had grade 1 tumor (58.3\%), 63 grade 2 (28.9\%), and 28 grade $3(12.8 \%)$. One hundred and sixty patients were
Table I. Expression of N-acetylgalactosaminyltransferase-6 (GalNAcT6) in relation to expression of cell adhesion molecules. The expression of GalNAc-T6 was found to be significantly correlated with the expression of E-cadherin in endometrial carcinomas.

\begin{tabular}{cccc}
\hline Expression & \multicolumn{2}{c}{ GalNAc-T6 expression, $\mathrm{n}$} & $p$-Value* \\
\cline { 2 - 3 } & $\begin{array}{c}\text { Positive } \\
\mathrm{n}=152\end{array}$ & $\begin{array}{c}\text { Negative } \\
\mathrm{n}=66\end{array}$ & \\
\hline E-Cadherin & & & \\
Negative & 83 & 46 & $0.038^{*}$ \\
Positive & 69 & 20 & \\
$\beta$-Catenin & & & \\
Negative & 75 & 39 & 0.186 \\
Positive & 77 & 27 & \\
\hline
\end{tabular}

*Mann-Whitney $U$-test.

Table II. Clinicopathological variables and $\mathrm{N}$-acetylgalactosaminyltransferase-6 (GalNAc-T6) expression. The expression of GalNAc-T6 was only statistically associated with histological grade.

\begin{tabular}{|c|c|c|c|}
\hline \multirow[t]{2}{*}{ Variable } & \multicolumn{2}{|c|}{ GalNAc-T6 } & \multirow[t]{2}{*}{$p$-Value* } \\
\hline & $\begin{array}{c}\text { Positive, } \mathrm{n} \\
\mathrm{n}=152\end{array}$ & $\begin{array}{c}\text { Negative, } n \\
n=66\end{array}$ & \\
\hline \multicolumn{4}{|l|}{ FIGO stage } \\
\hline Early (I, II) & 117 & 47 & 0.366 \\
\hline Advanced (III, IV) & 35 & 19 & \\
\hline \multicolumn{4}{|l|}{ Grade } \\
\hline 1 and 2 & 139 & 50 & $0.002^{*}$ \\
\hline 3 & 13 & 16 & \\
\hline \multicolumn{4}{|l|}{ Myometrial invasion } \\
\hline$<1 / 2$ & 109 & 51 & 0.394 \\
\hline$\geq 1 / 2$ & 43 & 15 & \\
\hline \multicolumn{4}{|l|}{ Vascular invasion } \\
\hline Negative & 102 & 40 & 0.356 \\
\hline Positive & 50 & 26 & \\
\hline \multicolumn{4}{|l|}{ Pelvic LN metastasis } \\
\hline Negative & 143 & 58 & 0.118 \\
\hline Positive & 9 & 8 & \\
\hline \multicolumn{4}{|l|}{ Cervical invasion } \\
\hline Negative & 135 & 59 & 0.901 \\
\hline Positive & 17 & 7 & \\
\hline \multicolumn{4}{|l|}{ Ovarian metastasis } \\
\hline Negative & 145 & 59 & 0.098 \\
\hline Positive & 7 & 7 & \\
\hline
\end{tabular}

LN: Lymph node; $<1 / 2$ :Tumor invasion limited to the inner half of the myometrium, FIGO stage 1 A. *Mann-Whitney $U$-test.

diagnosed with tumor invasion limited to the inner half of the myometrium (73.4\%). Vascular invasion was detected in 76 patients $(34.9 \%)$ but cervical involvement, ovarian and adnexa metastasis, pelvic lymph node metastasis were found in small number of patients, rating $11.0 \%$ (24 cases), $6.4 \%$ (14 cases) and $7.8 \%$ (17 cases) respectively (6). 

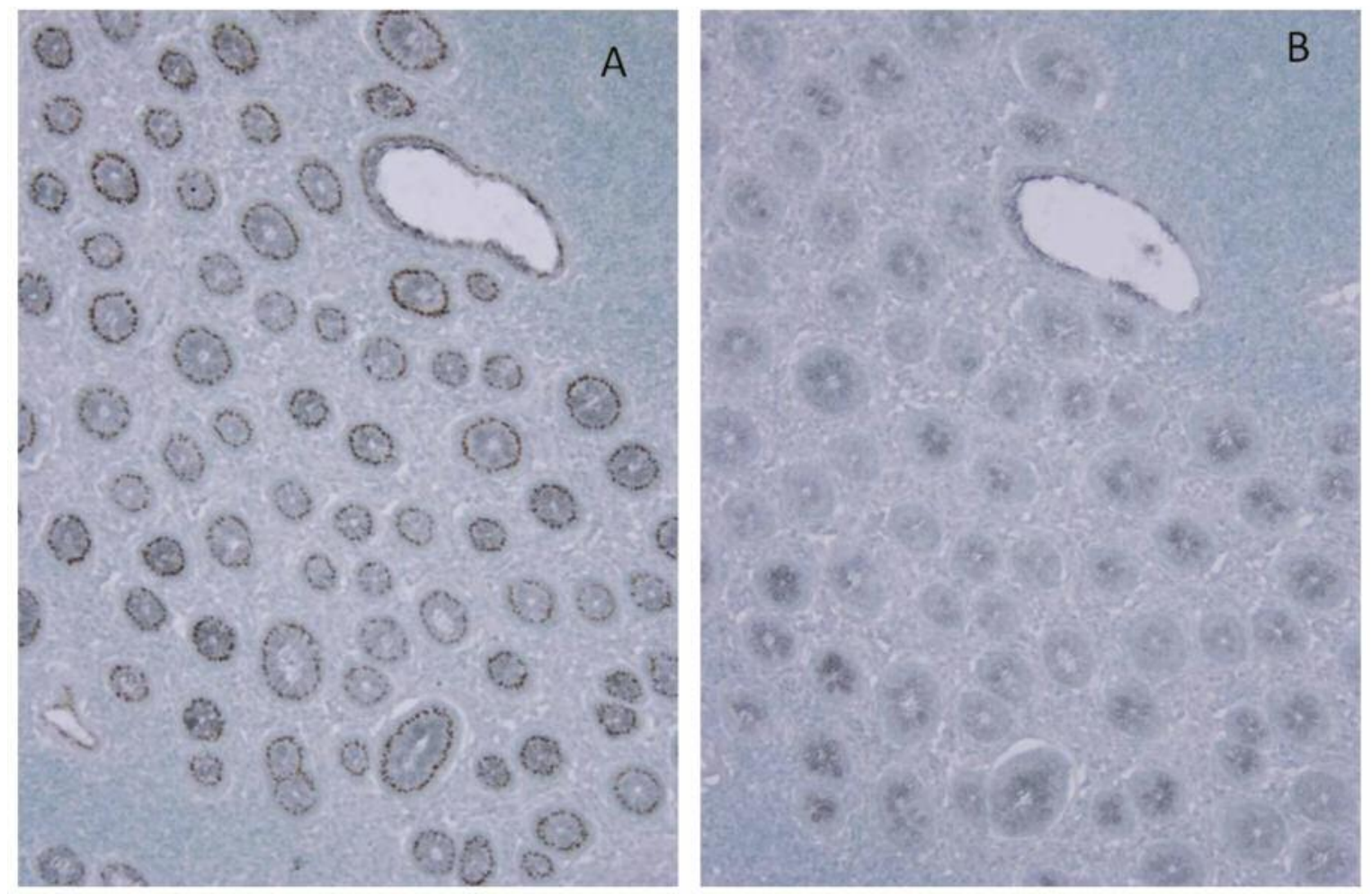

Figure 1. The specificity of a polyclonal antibody against N-acetylgalactosaminyltransferase-6 (GalNAc-T6) was tested by immunohistochemistry. A: Positive staining of intestine on incubation with a polyclonal antibody against GalNAc-T6. B: After incubation of this antibody with excess synthesized peptides of GalNAc-T6, positive immunostaining was almost abolished. Original magnification, $x 400$.

Immunohistochemical staining. One hundred and fifty-two cases $(69.7 \%)$ had positive cytoplasmic staining for GalNAcT6 and $66(30.3 \%)$ patients had negative staining. Eightynine patients $(40.8 \%)$ had positive membranous staining for E-cadherin, while 104 patients $(47.7 \%)$ had positive membranous staining for $\beta$-cadherin. The expression of GalNAc-T6 was found to be significantly positively correlated with the expression of E-cadherin $(p=0.038)$ (Table I).

The expression of GalNAc-T6 was statistical associated with histological grade of the tumor $(p=0.002)$ but not with the other features such as myometrial invasion $(p=0.394)$, vascular invasion $(p=0.356)$, and pelvic lymph nodes metastasis $(p=0.118)$ (Table II). On the other hand, Ecadherin expression was significantly positively correlated with myometrial invasion $(p=0.049)$ (Table III). $\beta$-Catenin expression was also significantly positively correlated with myometrial invasion $(p=0.004)$ and vascular invasion $(p=0.041)($ Table IV).

Prognostic significance. Univariate survival analysis showed that patients with well-differentiated tumors, superficial myometrial invasion, non-vascular invasion, non-lymph node metastasis, non-cervical invasion, non-ovarian metastasis, and positive GalNAc-T6 expression had better overall survival $(p<0.05)$. Positive E-cadherin and $\beta$-catenin expression were not significantly related to improved overall survival. Overall survival rate after 10 years appeared to be significantly higher in the group of patients exhibiting positive GalNAc-T6 staining (94.9\% compared with 77.8\%; $p=0.003)$. The multivariate analysis using the Cox proportional hazards model showed GalNAc-T6 expression to be an independent prognostic factor among the studied variables $(p=0.013)$ (Table V).

\section{Discussion}

To our knowledge, this is the first reported study to evaluate the correlation between immunohistochemical expression of GalNAc-T6, cell adhesion markers and clinical data for endometrial carcinoma. We previously reported that overexpression of GalNAc-T6 in patients with endometrial carcinoma was associated with lower invasion ability (6). In this study, we identified that expression of GalNAc-T6 is related to expression of E-cadherin and better histological grade of tumors. 
a

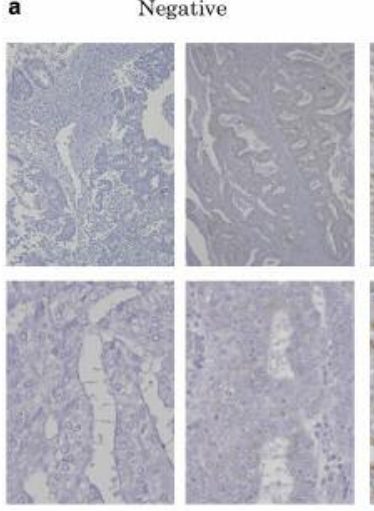

b

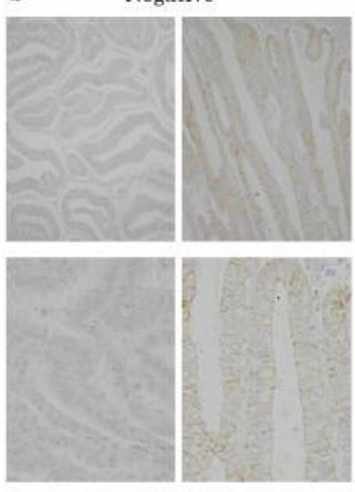

c Negative
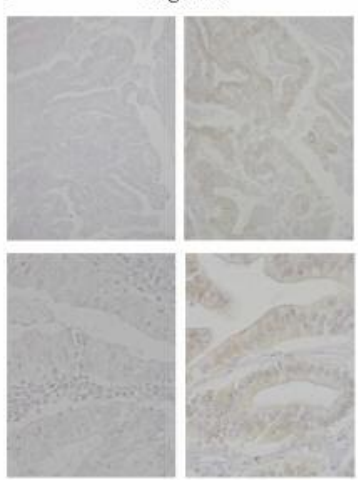

Figure 2. Images showing representative immunohistochemical expression of $N$-acetylgalactosaminyltransferase-6 (a), E-cadherin (b) and $\beta$-catenin $(c)$.

Cell adhesion factors are important in the structure and differentiation of cancer cells (21). E-Cadherin interacts with $\beta$-catenin and forms the cell adhesion complex linked to the cytoskeleton structure. Normal epithelial tissues show high expression of E-cadherin, but epithelial tumor cells often lose or reduce their E-cadherin expression (19). This phenomenon is purportedly related to tissue disorder and cellular dedifferentiation (19-22). On the other hand, increased production of cell adhesion complex including E-cadherin and $\beta$-catenin resulted in reduction of the invasiveness of pancreas and mammary cancer cells $(22,23)$. In this study, positive staining for GalNAc-T6 was significantly associated with better histological grade of tumors and positive staining for E-cadherin. Therefore, overexpression of GalNAc-T6 in patients with endometrial carcinoma might be related to characteristics of differentiation.

Positive staining for GalNAc-T6 appears to be an independent factor predicting better overall survival of patients with endometrial carcinoma $(p=0.003)$. However, a univariate survival analysis showed that the survival rate of patients with E-cadherin-positive and $\beta$-catenin-positive endometrial cancer was not significantly better than that of those with negative staining. Recently published reports indicated that knockdown of GalNAc-T6 led to EMT-like morphological changes of cancer cells, accompanied by the decrease of expression of cell adhesion molecules $(19,24,25)$. There is the possibility that abnormal GalNAc-T6 expression is involved in the cadherin switch in endometrial cancer.

In conclusion, our findings showed that the expression of GalNAc-T6 in endometrial carcinoma is significantly related to E-cadherin expression, and is an independent prognostic indicator of better overall survival. Our study was limited to immunohistochemical analysis, therefore we cannot rule-out the possibility that there is another mechanism by which GalNAc-T6 promotes endometrial carcinoma development and metastasis. More research is needed to support the hypothesis that GalNAc-T6 expression is related to cell-cell adhesion in the early phase of invasion in endometrial carcinoma.

\section{Conflilcts of Interest}

All Authors have no conflict of interest in regard to this study.

\section{References}

1 Yamagami W and Aoki D: Annual report of the Committee on Gynecologic Oncology, the Japanese Society of Obstetrics and Gynecology. J Obstet Gynaecol Res 41(2): 167-177, 2015.

2 Jarmal A, Bray F, Center MM, Ferlay J, Ward E and Forman D: Global cancer statistics. CA Can J Clin 61: 69-90, 2011.

3 Freire-de-Lima L and Gelfenbeyn K, Ding Y, Mandel U, Clausen $\mathrm{H}$, Handa $\mathrm{K}$ and Hakomori SI: Involvement of $O$-glycosylation defining oncofetal fibronection in epithelial-mesenchymal transition process. Proc Natl Acad Sci 108: 17690-17695, 2011.

4 Ding Y, Gelfenbeyn K, Freire-de-Lima L and Handa K and Hakomori SI: Induction of epithelial-mesenchymal transition with $O$-glycosylation of oncofetal fibronectin. FEBS Lett 586 : 1813-1820, 2012.

5 Alisson-Silva F and Freire-de L, Donadio JL, Lucena MC, Penha L, Sa-Diniz JN, Dias WB and Todeschini AR: Increase of $O$-glycosylated oncofetal fibronection in high glucose-induced epithelial-mesenchymal transition of cultured human epithelial cells. PLoS ONE 8: 60471-60477, 2013. 
Table III. Clinicopathological variables and E-cadherin expression. The expression of E-cadherin was only statistically related to myometrial invasion.

\begin{tabular}{|c|c|c|c|}
\hline \multirow[t]{2}{*}{ Variable } & \multicolumn{2}{|c|}{ E-Cadherin } & \multirow[t]{2}{*}{$p$-Value* } \\
\hline & $\begin{array}{c}\text { Positive, } \mathrm{n} \\
\mathrm{n}=89\end{array}$ & $\begin{array}{c}\text { Negative, } n \\
n=129\end{array}$ & \\
\hline \multicolumn{4}{|l|}{ FIGO stage } \\
\hline Early (I, II) & 69 & 95 & 0.515 \\
\hline Advanced (III, IV) & 20 & 34 & \\
\hline \multicolumn{4}{|l|}{ Grade } \\
\hline 1 and 2 & 81 & 108 & 0.120 \\
\hline 3 & 8 & 21 & \\
\hline \multicolumn{4}{|l|}{ Myometrial invasion } \\
\hline$<1 / 2$ & 59 & 108 & $0.049^{*}$ \\
\hline$\geq 1 / 2$ & 30 & 21 & \\
\hline \multicolumn{4}{|l|}{ Vascular invasion } \\
\hline Negative & 73 & 103 & 0.689 \\
\hline Positive & 16 & 26 & \\
\hline \multicolumn{4}{|l|}{ Pelvic LN metastasis } \\
\hline Negative & 80 & 121 & 0.291 \\
\hline Positive & 9 & 8 & \\
\hline \multicolumn{4}{|l|}{ Cervical invasion } \\
\hline Negative & 80 & 114 & 0.726 \\
\hline Positive & 9 & 15 & \\
\hline \multicolumn{4}{|l|}{ Ovarian metastasis } \\
\hline Negative & 85 & 119 & 0.336 \\
\hline Positive & 4 & 10 & \\
\hline
\end{tabular}

LN: Lymph node; <1/2:Tumor invasion limited to the inner half of the myometrium, FIGO stage $1 \mathrm{~A} . *$ Mann-Whitney $U$-test.
Table IV. Clinicopathological variables and $\beta$-catenin expression. The expression of $\beta$-catenin was statistically significantly related to myometrial and vascular invasion.

\begin{tabular}{|c|c|c|c|}
\hline \multirow[t]{2}{*}{ Variable } & \multicolumn{2}{|c|}{$\beta$-Catenin } & \multirow[t]{2}{*}{$p$-Value* } \\
\hline & $\begin{array}{c}\text { Positive, } n \\
n=104\end{array}$ & $\begin{array}{l}\text { Negative, } \mathrm{n} \\
\mathrm{n}=114\end{array}$ & \\
\hline \multicolumn{4}{|l|}{ FIGO stage } \\
\hline Early (I, II) & 72 & 92 & 0.051 \\
\hline Advanced (III, IV) & 32 & 22 & \\
\hline \multicolumn{4}{|l|}{ Grade } \\
\hline 1 and 2 & 93 & 96 & 0.259 \\
\hline 3 & 11 & 18 & \\
\hline \multicolumn{4}{|l|}{ Myometrial invasion } \\
\hline$<1 / 2$ & 67 & 93 & $0.004 *$ \\
\hline$\geq 1 / 2$ & 37 & 21 & \\
\hline \multicolumn{4}{|l|}{ Vascular invasion } \\
\hline Negative & 78 & 98 & $0.041^{*}$ \\
\hline Positive & 26 & 16 & \\
\hline \multicolumn{4}{|l|}{ Pelvic LN metastasis } \\
\hline Negative & 92 & 109 & 0.050 \\
\hline Positive & 12 & 5 & \\
\hline \multicolumn{4}{|l|}{ Cervical invasion } \\
\hline Negative & 92 & 102 & 0.812 \\
\hline Positive & 12 & 12 & \\
\hline \multicolumn{4}{|l|}{ Ovarian metastasis } \\
\hline Negative & 94 & 110 & 0.067 \\
\hline Positive & 10 & 4 & \\
\hline
\end{tabular}

LN: Lymph node; <1/2:Tumor invasion limited to the inner half of the myometrium, FIGO stage $1 \mathrm{~A} . *$ Mann-Whitney $U$-test.

Table V. Univariate and multivariate analyses of prognostic variables for overall survival in endometrial carcinoma.

\begin{tabular}{|c|c|c|c|c|c|c|}
\hline \multirow[b]{2}{*}{ Variable (Ref $v s$. predictor) } & \multicolumn{3}{|c|}{ Univariate analysis } & \multicolumn{3}{|c|}{ Multivariate analysis } \\
\hline & HR & $95 \% \mathrm{CI}$ & $p$-Value & HR & $95 \% \mathrm{CI}$ & $p$-Value \\
\hline Histological grade: 1 and 2 vs. 3 & 6.032 & $2.378-15.299$ & 0.001 & 2.106 & $0.571-7.768$ & 0.264 \\
\hline Myometrial invasion: $<1 / 2$ vs. $\geq 1 / 2$ & 16.041 & $4.624-55.644$ & 0.001 & 11.595 & $2.56-52.520$ & $0.001 *$ \\
\hline Vascular invasion: Negative $v s$. positive & 7.282 & $2.392-22.172$ & 0.001 & 1.466 & $0.360-5.975$ & 0.593 \\
\hline LN metastases: Negative $v s$. positive & 11.322 & $4.457-28.763$ & 0.001 & 1.497 & $0.386-5.805$ & 0.560 \\
\hline Cervical invasion: Negative $v s$. positive & 3.426 & $1.221-9.614$ & 0.019 & 0.983 & $0.292-3.304$ & 0.977 \\
\hline Ovarian metastasis: Negative $v s$. positive & 4.108 & $1.350-12.504$ & 0.013 & 1.254 & $0.313-5.024$ & 0.749 \\
\hline GalNAc-T6: Negative $v s$. positive & 0.263 & $0.102-0.678$ & 0.006 & 0.270 & $0.096-0.757$ & $0.013 *$ \\
\hline E-Cadherin: Negative $v s$. positive & 0.726 & $0.272-1.935$ & 0.522 & & & \\
\hline$\beta$-Catenin: Negative $v s$. positive & 0.843 & $0.332-2.137$ & 0.719 & & & \\
\hline
\end{tabular}

Ref: Referent; HR: hazard ratio; CI: confidence interval; LN: lymph node; GalNAc-T6: $N$-acetylgalactosaminyltransferase-6.

6 Nguyen TT, Kurita T, Koi C, Murakami M, Kagami S, Hachisuga T, Hisaoka M, Morimoto Y and Izumi H: GalNAcT6 in the relationship with invasion ability of endometrial carcinomas and prognostic significance. Am J Cancer Res 7(5): 1188-1197, 2017.

7 Shanfeng Y, Britt MH, Zhongze Li, Gregory DS, Jiang G, Jan LF and Marc SE: Epithelial-Mesenchymal expression phenotype of primary melanoma and matched metastases and relationship with overall survival. Anticancer Res 36: 6449-6456, 2016.

8 Sakuragi N, Nishiya M, Ikeda K, Ohkouch T, Furth EE, Hareyama H, Satoh C and Fujimoto S: Decreased E-cadherin expression in endometrial carcinoma is associated with tumor dedifferentiation and deep myometrial invasion. Gynecol Oncol 53: 183-189, 1994. 
9 Shih HC, Shiozawa T, Miyamoto T, Kashima H, Feng YZ, Kurai M, Konishi I : Immunohistochemical expression of E-cadherin and $\beta$-catenin in the normal and malignant human endometriun: an inverse correlation between E-cadherin and nuclear $\beta$-catenin expression. Anticancer Res 24: 3843-3850. 2011.

10 Brockhausen I: Mucin-type $O$-glycans in human colon and breast cancer: glycodynamics and functions. EMBO Rep 7(6): 599-604, 2006.

11 Fuster MM and Esko JD: The sweat and sour of cancer: glycans as novel therapeutic targets. Nature 5: 526-542, 2005.

12 Nakamura K, Yamashita H, Sawaki H, Nakayama N, Kawamata $\mathrm{H}$, Nishiyama A, Narimatsu $\mathrm{H}$ and Watanabe $\mathrm{M}$ : Aberrant methylation of GCNT2 is tightly related to lymph node metastasis of primary CRC. Anticancer Res 35: 1411-1421, 2015.

13 Clausen H and Eric BP: A family of UDP-GalNac: polypeptide $\mathrm{N}$-acetylgalactosaminyl-transferase control the initiation of mucintype $O$-linked glycosylation. Glycobiol 6(6): 635-646, 1996.

14 Li Z, Yamada S, Wu Y, Wang KY, Liu YP, Uramoto H, Kohno $\mathrm{K}$ and Sasaguri Y: Polypeptide $N$-acetylgalactosaminyltransferase- 6 expression independently predicts poor overall survival in patients with lung adenocarcinoma after curative resection. Oncotarget 7(34): 54463-54473, 2016.

$15 \mathrm{Li} \mathrm{Z}$, Yamada S, Inenaga S, Imamura T, Wu Y, Wang KY, Shimajiri S, Nakano R, Izumi H, Kohno K and Sasaguri Y: Polypeptide $N$-acetylgalactosaminyltransferase 6 expression in pancreatic cancer is an independent prognostic factor indicating better overall survival. Br J Cancer 104: 1882-1889, 2011.

16 Berois N, Mazal D, Ubillos L, Trajtenberg F, Nicolas A, SastreGarau X and Magdelenat $\mathrm{H}$ and Osinaga E: UDP-N-acetyl-Dgalactosamine: polypeptide $N$-acetylgalactosaminyltransferase- 6 as a new immunohistochemical breast cancer marker. J Histochem Cytochem 54(3): 317-328, 2006.

17 Kitada S, Yamada S, Kuma A, Ouchi S, Tasaki T, Nabeshima A, Noguchi H, Wang KY, Shimajiri S, Nakano R, Izumi H, Kohno $\mathrm{K}$, Matsumoto $\mathrm{T}$ and Sasaguri $\mathrm{Y}$ : Polypeptide $\mathrm{N}$ acetylgalactosaminyl transferase 3 independently predicts highgrade tumours and poor prognosis in patients with renal cell carcinomas. Br J Cancer 109(2): 472-478, 2013.

18 Harada Y, Izumi H, Noguchi H, Kuma A, Kawatsu Y, Kimura T, Kitada S, Uramoto H, Wang KY, Sasaguri Y, Hijioka H, Miyawaki A, Oya R, Nakayama T, Kohno K and Yamada S: Strong expression of polypeptide $N$-acetylgalactosami-nyltransferase 3 independently predicts shortened disease-free survival in patients with early stage oral squamous cell carcinoma. Tumour Biol 37(1): 1357-1368, 2016.
19 Tarhan YE, Kato T, Jang M, Haga Y, Ueda K, Nakamura Y and Park JH: Morphological changes, cadherin switching, and growth suppression in pacreatic cancer by GALNT6 knockdown. Neoplasia 18(5): 265-272, 2016.

20 Lewin SN. Revised FIGO staging system for endometrial cancer Clin Obstet Gynecol 54(2): 215-218, 2011.

21 Oyama T, Kanai Y, Ochiai A, Akimoto S, Oda T, Yanagihara K, Nahafuchi A, Tsukita A, Shibamoto A, Ito F, Takeuchi M, Matsuda $\mathrm{H}$ and Hirohashi S: A truncated $\beta$-catenin disrupts the interaction between E-cadherin and $\alpha$-catenin: A cause of loss of intercellular adhesiveness in human cancer cell lines. Cancer Res 54(23): 6282-6297, 1994.

22 Paredes J, Figueiredo J, Albergaria A, Oliverira P, Carvalho J, Ribeiro AS, Caldeira J, Costa AM, Simoes-Correria J and Oliveira MJ: Epithelial E and P-cadherins: role and clinical significance in cancer. Biochim Biophy Acta 1826(2): 297-311, 2012.

23 Taniguchi K, Nakagawa H, Hosokawa M, Nakamura T, Eguchi $\mathrm{H}$, Ohigashi $\mathrm{H}$, Ishikawa $\mathrm{O}$, Katagiri $\mathrm{T}$ and Nakamura $\mathrm{Y}$ : Overexpressed P-cadherin/CHD3 promotes motility of pancreatic cancer cells by interacting with p120ctn and activating rho-family GTPases. Cancer Res 65(8): 3092-3099, 2005.

24 Park JH, Nishidate T, Kijima K, Ohashi T, Takegawa K, Fujikane T, Hirata K, Nakamura Y and Katagiri T: Critical roles of mucin 1 glycosylation by transactivated polypeptide $\mathrm{N}$ acetylgalactosaminyltransferase 6 in mammary carcinogenesis. Cancer Res 70(7): 2759-2769, 2010.

25 Park JH, Katagiri T, Chung S, Kijima K and Nakamura Y: Polypeptide $N$-acetylgalactosaminyltransferase 6 disrupts mammary acinar morphogenesis through $O$-glycosylation of fibronectin. Neoplasia 13(4): 320-326, 2011.
Received May 2, 2017

Revised May 22, 2017

Accepted May 23, 2017 